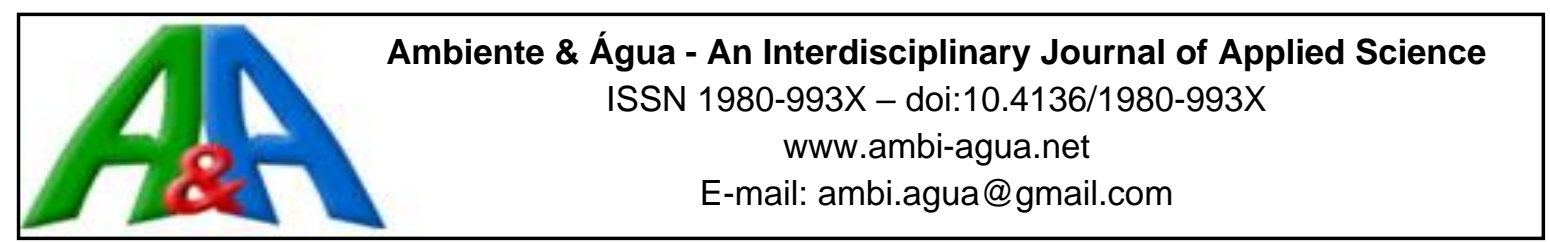

\title{
Ocorrência de um molusco invasor (Melanoides tuberculata, Müller, 1774), em diferentes sistemas aquáticos da bacia hidrográfica do Rio Sorocaba, SP, Brasil
}

\author{
doi:10.4136/ambi-agua.1971
}

Received: 25 Jul. 2016; Accepted: 21 Jun. 2017

\author{
Cecília Maria de Paula ${ }^{1}$; Adriane Almeida Vaz'; \\ Ariane Almeida Vaz ${ }^{1}$; Gisele Pires Pelizari ${ }^{1}$; \\ Heidi Marcela Suarez Robayo²; Thaís Deluno Garcia'; \\ Daniely Avelino'; Giuliano Grici Zacarin ${ }^{1}$; \\ Welber Senteio Smith ${ }^{1^{*}}$ \\ ${ }^{1}$ Universidade Paulista (UNIP), Sorocaba, SP, Brasil \\ Laboratório de Ecologia Estrutural e Funcional \\ ${ }^{2}$ Universidade de São Paulo (USP), São Carlos, SP, Brasil \\ Programa de Pós-Graduação em Ciências da Engenharia Ambiental, \\ Centro de Recursos Hídricos e Ecologia Aplicada \\ *Autor correspondente: e-mail: welber_smith@uol.com.br, \\ ceci_mdpa@hotmail.com, adriane_almvaz@hotmail.com, ariane_almvaz@hotmail.com, \\ giselepelzbio@gmail.com,hmsr_@hotmail.com,tata_delunoGarcia@hotmail.com, \\ danielyavelino@uol.com.br, gzacarin@yahoo.com.br
}

\section{RESUMO}

No Brasil e no estado de São Paulo existem poucos levantamentos sobre a presença do gastrópode Melanoides tuberculata (Müller, 1774). Os moluscos invasores representam uma importante ameaça às espécies nativas, em consequência da sua agressividade na competição pelo uso dos habitats e dos diferentes recursos. Melanoides tuberculata é originário da Ásia e África e a sua introdução no Brasil data da década de 60. Estudos recentes ressaltam a importância de realizar novas pesquisas sobre a distribuição espacial desta espécie e seu possível impacto à biodiversidade. O presente estudo documenta a ocorrência da espécie Melanoides tuberculata na bacia hidrográfica do rio Sorocaba, sua abundância e distribuição espacial em 5 sistemas aquáticos em diferentes estágios de conservação. As amostragens foram realizadas nos meses de junho de 2013 até agosto de 2014, onde foram registrados 522 indivíduos, dos quais $67 \%$ foram encontrados no Rio Verde, localizado na zona de amortecimento da Floresta Nacional de Ipanema. Os resultados mostraram que esta espécie pode estar amplamente distribuída na bacia principalmente em locais com substrato arenoso, mata ripária degradada e sujeito a lançamento de esgoto. Deve ser ressaltada ainda a correlação com valores de temperatura da água mais altos, o que pode estar ligado à ausência de sombreamento propiciado pela mata ripária. O controle na proliferação desta espécie, deve ser realizado pela melhoria da qualidade ambiental do ambiente aquáticos uma vez que a erradicação se torna inviável por remoção física.

Palavras-chave: caramujo aquático, córregos, espécies introduzidas, impactos, invasão biológica. 


\title{
Occurrence of the invasive mollusc Melanoides tuberculata (Müller, 1774) in different aquatic systems of the Sorocaba River Basin, Brazil
}

\begin{abstract}
There are few surveys in Brazil of the presence of the gastropod Melanoides tuberculate (Müller, 1774). Invasive mollusks pose a major threat to native species as a result of their aggressive competition for habitat use and various resources. Native to Asia and Africa, Melanoides tuberculata was introduced to Brazil in the 1960's. Recent studies have discussed the importance of conducting further research on the spatial distribution of this species and its possible impact on biodiversity. Sampling was conducted from June 2013 to August 2014, and a total of 522 individuals was recorded; $67 \%$ of these were found in the Rio Verde River, located in the buffer zone of the Ipanema National Forest. The results showed that this species may be widely distributed in the basin, especially in places with sandy soil, degraded riparian zones, and areas where sewage is discharged. It is important to note the correlation of the distribution with higher water temperatures, which may be related to the absence of shading of riparian forests. Control of the proliferation of this species, which has a high attack potential, must be accomplished by improving quality of the aquatic environment, since eradication by physical removal is unfeasible.
\end{abstract}

Keywords: biological invasion, freshwater snails, impacts, introduced species, stream.

\section{INTRODUÇÃO}

Com a crescente globalização e o consequente aumento do fluxo de mercadorias e pessoas, espécies aquáticas e terrestres têm sido transferidas acidental ou deliberadamente para áreas fora de sua distribuição geográfica natural, onde podem encontrar condições ambientais adequadas para sua sobrevivência, tornando-se abundantes e dominantes (Everett, 2000; Rosa et al., 2010). Isso é agravado, devido à degradação ambiental dos ecossistemas (Pimentel et al., 2001), que muitas vezes favorece as invasoras.

O fluxo natural das águas auxilia na dispersão dos organismos invasores em sistemas aquáticos (Okumura, 2006). Por isso, os ambientes de água doce estão altamente sujeitos às invasões de organismos exóticos já que apresentam grande facilidade para a dispersão pelo próprio fluxo d'água (Welcomme, 1988). Além disso, outros mecanismos facilitam a sua dispersão, como algumas espécies de aves que podem levar moluscos em seus pés ou plumagem, ou mesmo através do canal alimentar ou incrustações em embarcações, que pode ser uns dos principais vetores para as invasões biológicas (Silva e Barros, 2011).

Em 2006 o Ministério do Meio Ambiente produziu um "Informe Nacional sobre espécies exóticas invasoras no Brasil”. O resultado foi uma lista de 543 espécies invasoras no território nacional, sendo documentadas 49 espécies para águas continentais, sendo 4 espécies de moluscos. A Secretaria do Meio Ambiente do estado de São Paulo constituiu um Grupo de Trabalho (Resoluções SMA 033/2009 e 050/2009. São Paulo, 2009) com a incumbência de elaborar proposta de estratégia estadual para espécies exóticas e invasoras. O documento insere a espécie Melanoides tuberculata na categoria I, a qual indica as espécies que o uso; posse ou domínio; transporte, soltura ou translocação; propagação (cultivo, criação ou qualquer forma de reprodução) e comércio; doação ou aquisição não podem ser realizados. Exceções configuram o uso de espécimes mortos (por exemplo, consumo ou uso como matéria prima) ou para pesquisa científica. Em seu anexo I o documento se refere a ocorrência da espécie em ambientes lênticos na Bacia do Baixo Tietê e Tiete-Batalha.

Acredita-se que o mecanismo mais provável de introdução da espécie alvo do presente estudo foi a libertação desses organismos antes usados em aquários, como sugerido por Vaz et 
al. (1986). Deve ser salientado, que rápidos processos de invasão também estão em andamento por outras espécies de moluscos, como o bivalve asiático Corbicula fluminea e o mexilhão dourado Limnoperna fortunei (Rocha et al., 2011).

O Melanoides tuberculata (Muller, 1774) é um gastrópode nativo do norte e leste da África e sudeste da Ásia, sendo observado pela primeira vez no Brasil na cidade de Santos, SP (Vaz et al., 1986; Abílio, 1997). Desde então tem sido encontrado em diversos estados brasileiros (Fernandez et al., 2003; Thiengo et al., 2007), como Goiás (Rocha-Miranda e Martins-Silva, 2006.), Minas Gerais (Pinto e Melo, 2010; Souza et al., 2006), Brasília (PaulaAndrade et al., 2012), Rio Grande do Norte (Santos e Eskinazi-Sant'Anna, 2010), Paraíba (Abílio e Watanabe, 1998), Pernambuco (Souza et al., 2010) e Espírito Santo (Vaz et al., 1986).

No estado de São Paulo além de Santos, há também relatos dessa espécie desde 1980 em Ribeirão Preto (Vaz et al., 1986). Além disso, foi uma das espécies dominantes encontrada no reservatório de Salto Grande em Americana, SP (Pamplim et al., 2005) e nas represas do médio e baixo rio Tietê (Suriani et al., 2007; Rocha et al., 2011). Com a grande ocorrência deste organismo no Brasil e especialmente no estado de São Paulo, é de grande importância fornecer informações da ocorrência e da sua distribuição espacial, sendo informações úteis para o controle da sua dispersão. Portanto, o objetivo deste trabalho foi descrever a ocorrência do gastrópode invasor Melanoides tuberculata, sua abundância e distribuição espacial em diferentes sistemas aquáticos pertencentes a bacia hidrográfica do Rio Sorocaba, SP, Brasil, investigando fatores que favoreçam a sua invasão.

\section{MATERIAL E MÉTODOS}

A bacia do Rio Sorocaba situa-se na sub-área conhecida por Médio Tietê, da qual é a segunda maior bacia hidrográfica (Figura 1). Está localizada no trecho superior do Planalto Atlântico e parte na Depressão Periférica, nos afloramentos do grupo Tubarão na bacia sedimentar do Paraná, abrangendo 22 municípios (Smith, 2003).

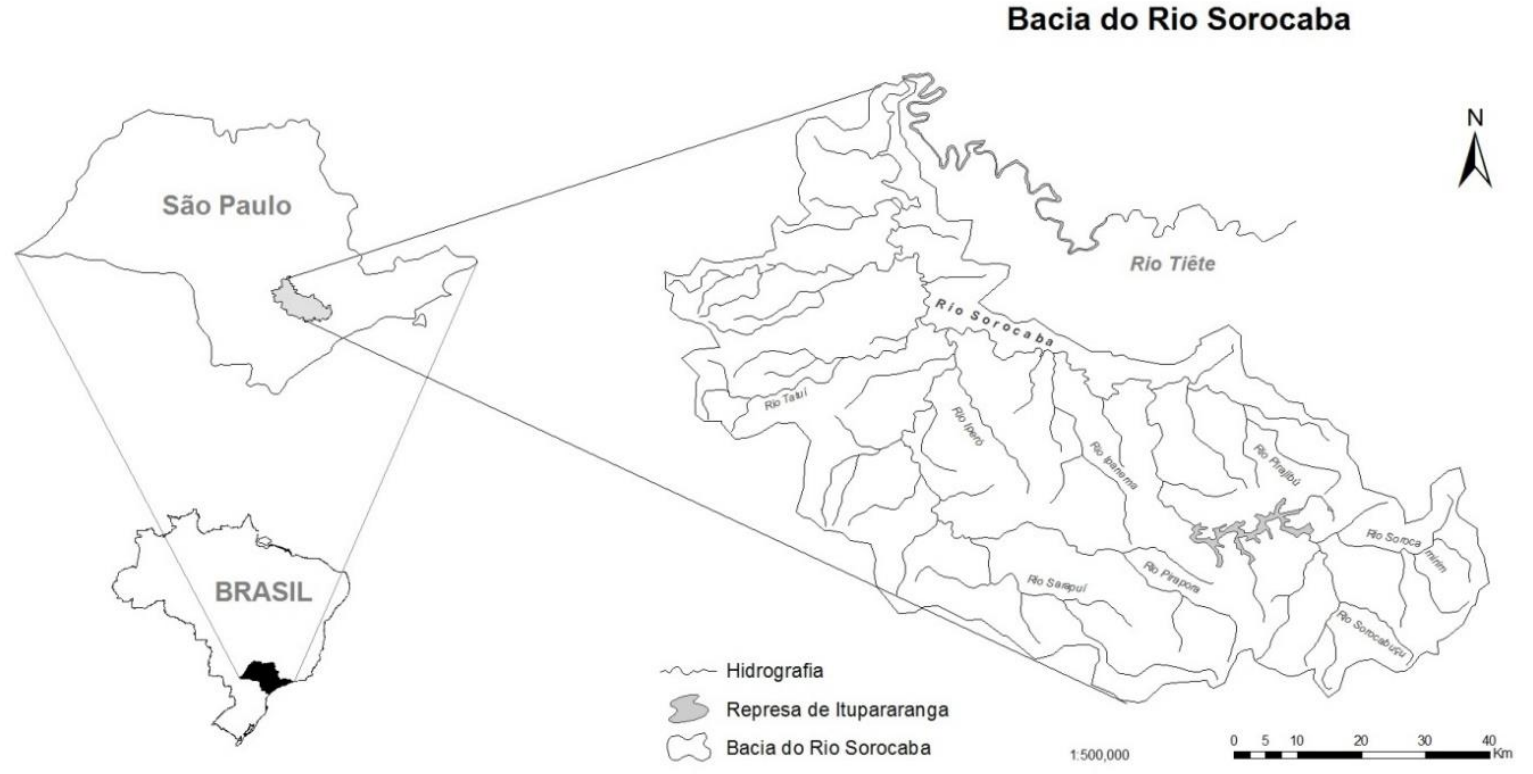

Figura 1. Bacia hidrográfica do rio Sorocaba, SP, Brasil.

Foram inventariados 5 corpos d'água (Figura 2) totalizando 24 pontos, baseado em informações prévias da ocorrência de moluscos invasores. Nas distintas áreas foram realizadas diferentes campanhas entre os meses de junho de 2013 a agosto de 2014, com uma 
campanha no Ribeirão do Ferro (fe1, fe2 e fe3) no mês de agosto de 2014, duas campanhas no rio Ipanema (ip1, ip2, ip3, ip4 e ip5) nos meses de janeiro e agosto de 2014, quatro campanhas, nos meses de junho a dezembro de 2013 no rio Verde (ve1, ve2, ve3, ve4, ve5, ve6 e ve7), quatro campanhas no córrego Campininha (ca1, ca2, ca3 e ca4) entre os meses de janeiro e julho de 2014 e cinco campanhas nos meses de abril a agosto de 2014 no Córrego Itaguaraguaiaú localizado no Parque Chico Mendes (ch1, ch2, ch3, ch4 e ch5).

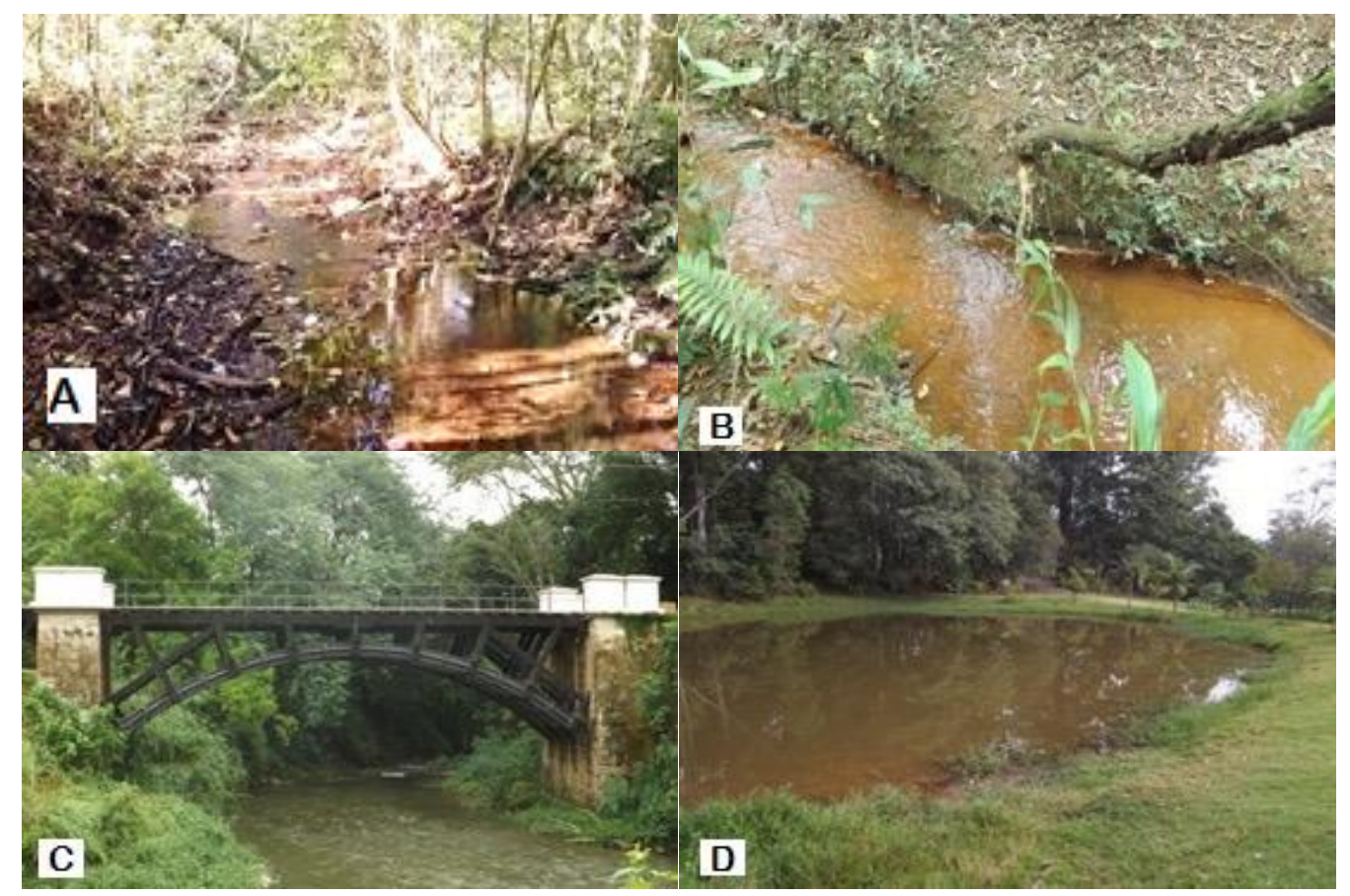

Figura 2. Corpos d' água estudados: (A) Ribeirão do Ferro; (B) Rio Verde; (C) Rio Ipanema; (D) Barramento do Córrego Itaguaraguaiaú.

Para a caracterização ambiental dos sistemas aquáticos foram mensuradas as seguintes variáveis limnológicas: $\mathrm{pH}$, condutividade elétrica, oxigênio dissolvido e temperatura da água, usando uma sonda multi-parâmetro da marca Oakton 600 Series Water proof Portable Meters. Além disso, foi obtido para cada ponto a profundidade, largura, tipo de substrato e a condição da mata ripária (degradada ou conservada). As amostragens foram feitas com uma rede "d", com malha de 500 micras. Após a coleta, as amostras foram transferidas em sacos plásticos contendo água do próprio local para triagem dos organismos vivos, facilitando desta forma a visualização. Todas as coletas foram realizadas através da autorização para atividades com finalidade cientifica SISBIO número 43708-2.

A triagem dos organismos foi realizada no laboratório de Ecologia Estrutural e Funcional da Universidade Paulista, Campus Sorocaba. O material recolhido foi lavado em peneira com malha de 500 micras e colocado em bandeja branca em local bem iluminado. A identificação dos gastrópodes foi feita através das características das conchas utilizando as chaves taxonômicas de Barbosa (1995) e Souza e Lima (1997).

Para a análise dos dados, a partir de uma matriz de correlação, foi utilizada uma análise de componentes principais (ACP), cuja técnica de ordenação visa arranjar as amostras em um menor número de dimensões, enfatizando os maiores padrões de variações em suas respostas, produzindo máxima informação sobre os padrões e similaridades ecológicas (Digby e Kempton, 1987). Esta técnica reduz a dimensão dos dados sem perder informações, dando ênfase aos maiores padrões de variações. Os melhores resultados são obtidos quando as variáveis originais são altamente correlacionadas (Manly, 1986). A ACP auxiliou na geração de hipóteses, utilizando os dados ambientais e a abundância de indivíduos obtida em cada 
ponto de coleta (Digby \& Kempton, 1987). O procedimento consistiu em: (1) definir os novos eixos de direção e comprimento pelo cálculo dos autovetores e autovalores da matriz de correlação entre as variáveis limnológicas e abundância; (2) calcular a correlação entre as variáveis e os eixos principais para a formação dos respectivos eixos; (3) calcular as coordenadas dos pontos observados nos novos eixos. Foram desconsiderados os valores inferiores a 0,4, para analisar apenas as maiores contribuições, com a finalidade de evitar erros nas interpretações dos resultados.

\section{RESULTADOS E DISCUSSÃO}

A comparação dos sistemas aquáticos estudados mostrou diferenças nas características ambientais avaliadas. O Ribeirão do Ferro (Fe) localizado na Floresta nacional de Ipanema apresentou largura entre um e dois metros e profundidades sempre inferiores a um metro. $\mathrm{O}$ substrato é predominantemente rochoso e arenoso com material orgânico de origem alóctone como folhas, galhos e outros. Suas águas possuem alta transparência, apresentando boa oxigenação. Apresentam características similares nos três pontos caracterizados, sendo estes completamente recobertos por densa vegetação nativa e baixa vegetação introduzida, com pouca interferência antrópica. O rio Ipanema (Ip) também localizado na Floresta Nacional de Ipanema, teve 5 pontos amostrados apresentando baixa profundidade, largura acima de 3 metros, substrato pedregoso, presença de galhos no leito e vegetação ripária relativamente abundante. O Rio Verde (Ve) localizado no município de Araçoiaba da Serra foi avaliado em 7 pontos de coleta. Apresentou baixa profundidade, largura entre 1 e 2 metros, substrato predominantemente arenoso, provavelmente procedente de assoreamento devido a antropização de suas margens, tendo a sua mata ripária degradada (Tabela 1).

Tabela 1. Pontos de amostragem e caracterização dos ambientes estudados.

\begin{tabular}{|c|c|c|c|c|}
\hline Código & Denominação & Munícipio & Localização Geográfica & Caracterização \\
\hline $\mathrm{Fe}$ & $\begin{array}{l}\text { Riacho Ribeirão } \\
\text { do Ferro }\end{array}$ & Iperó & $\begin{array}{l}23^{\circ} 25^{\prime} 58,14^{\prime} \mathrm{S} \\
47^{\circ} 37^{\prime} 27,27^{\prime} \mathrm{W}\end{array}$ & $\begin{array}{l}\text { Mata } \\
\text { conservada, sedimento } \\
\text { de rocha e areia, } \\
\text { sistema lótico }\end{array}$ \\
\hline Ip & Rio Ipanema & Araçoiaba da Serra & $\begin{array}{l}23^{\circ} 42^{\prime} 01,58^{\prime} \mathrm{S} \\
47^{\circ} 59^{\prime} 33,11^{\prime \prime} \mathrm{W}\end{array}$ & $\begin{array}{l}\text { Mata ciliar } \\
\text { conservada, sedimento } \\
\text { de rocha e areia, } \\
\text { sistema lótico }\end{array}$ \\
\hline $\mathrm{Ve}$ & Rio Verde & Araçoiaba da Serra & $\begin{array}{l}23^{\circ} 44^{\prime} 40,67^{\prime} \mathrm{S} \\
47^{\circ} 59^{\prime} 11,38^{\prime \prime} \mathrm{W}\end{array}$ & $\begin{array}{l}\text { Mata ciliar degradada, } \\
\text { sedimento de areia, } \\
\text { sistema lótico }\end{array}$ \\
\hline $\mathrm{Ca}$ & $\begin{array}{l}\text { Córrego da } \\
\text { Campininha }\end{array}$ & Sorocaba & $\begin{array}{l}23^{\circ} 22^{\prime} 35^{\prime \prime} \mathrm{S} \\
47^{\circ} 28^{\prime} 03^{\prime \prime} \mathrm{W}\end{array}$ & $\begin{array}{l}\text { Mata ciliar degradada, } \\
\text { sedimento de lodo e } \\
\text { areia, sistema lótico }\end{array}$ \\
\hline $\mathrm{Ch}$ & $\begin{array}{l}\text { Córrego } \\
\text { Itaguaraguaiaú }\end{array}$ & Sorocaba & $\begin{array}{l}23^{\circ} 30^{\prime} 06^{\prime \prime S} \\
47^{\circ} 27^{\prime} 29^{\prime \prime} \mathrm{W}\end{array}$ & $\begin{array}{l}\text { Mata ciliar degradada, } \\
\text { sedimento de lodo e } \\
\text { areia, sistema lêntico }\end{array}$ \\
\hline
\end{tabular}

O córrego da Campininha $(\mathrm{Ca})$ apresentou largura e profundidades sempre inferiores a dois metros, substrato predominantemente arenoso, apresentando folhas e galhos depositados no leito. Suas águas são turvas, apresentando baixos valores de oxigenação em alguns trechos. Os pontos amostrados se mostraram alterados, e não conservam suas características naturais principalmente devido à degradação da vegetação ripária desencadeada pela monocultura de eucalipto. O córrego Itaguaraguaiaú localizado no parque Natural Chico Mendes (Ch) 
apresenta-se represado, possuindo mata riparia em estado inicial, com predominância de eucaliptos nas áreas marginais, substrato lodoso e arenoso (Tabela 1).

Os resultados relativos às variáveis físicas e químicas são apresentados na Tabela 2. De forma geral, os $\mathrm{pH}$ de todos os pontos apresentaram valores semelhantes, estando enquadrados no CONAMA no 357/2005 que 6 a 9. Os valores médios de condutividade variaram entre $172,55 \mu \mathrm{S} / \mathrm{cm}$ e $183,68 \mu \mathrm{S} / \mathrm{cm}$ no Ribeirão do Ferro, 121,5 $\mu \mathrm{S} / \mathrm{cm}$ e 123,9 $\mu \mathrm{S} / \mathrm{cm}$ no Rio Ipanema, $81,55 \mu \mathrm{S} / \mathrm{cm}$ e $84,3 \mu \mathrm{S} / \mathrm{cm}$ no Rio Verde, $69,0 \mu \mathrm{S} / \mathrm{cm}$ e $79,5 \mu \mathrm{S} / \mathrm{cm}$ no Córrego da Campininha e 82,7 $\mu \mathrm{S} / \mathrm{cm}$ e $88,2 \mu \mathrm{S} / \mathrm{cm}$ no Parque Chico Mendes. Apesar do CONAMA $n^{\circ} 357 / 2005$ não estipular valores para condutividade, os valores encontrados apresentaram-se altos quando comparados a outros sistemas aquáticos similares.

Tabela 2. Médias das variáveis físicas e químicas da água nos diferentes sistemas aquáticos estudados.

\begin{tabular}{|c|c|c|c|c|c|c|c|}
\hline Local & Ponto & $\mathrm{pH}$ & $\begin{array}{c}\text { Condutividade } \\
\mu \mathrm{S} / \mathrm{cm}\end{array}$ & $\begin{array}{c}\text { Oxigênio } \\
\text { Dissolvido } \mathrm{mg} \mathrm{L}^{-1}\end{array}$ & Temperatura ${ }^{\circ} \mathrm{C}$ & $\begin{array}{l}\text { Profundidade } \\
\text { metros }\end{array}$ & Largura metros \\
\hline \multirow{3}{*}{$\begin{array}{l}\text { Ribeirão do } \\
\text { Ferro }\end{array}$} & fe1 & 6,56 & 183,68 & 6,32 & 17,48 & 0,28 & 3,34 \\
\hline & $\mathrm{fe} 2$ & 6,50 & 183,06 & 6,44 & 17,42 & 0,22 & 2,40 \\
\hline & fe 3 & 6,20 & 172,55 & 6,21 & 17,56 & 0,48 & 2,45 \\
\hline \multirow{5}{*}{ Rio Ipanema } & ip1 & 6,67 & 123,60 & 12,75 & 18,20 & 0,80 & 2,30 \\
\hline & ip2 & 6,46 & 123,90 & 7,00 & 19,10 & 0.75 & 1,80 \\
\hline & ip3 & 7,01 & 122,40 & 6,41 & 17,80 & 0.85 & 3,50 \\
\hline & ip4 & 6,57 & 121,50 & 8,20 & 16,40 & 0,70 & 2,50 \\
\hline & ip5 & 6,78 & 123,50 & 6,34 & 18,40 & 0,26 & 1,80 \\
\hline \multirow{7}{*}{ Rio Verde } & ve1 & 6,90 & 81,55 & 9,10 & 24,70 & 0,64 & 2,77 \\
\hline & ve2 & 6,80 & 82,55 & 9,25 & 24,50 & 0,95 & 1,65 \\
\hline & ve3 & 7,00 & 82,55 & 8,75 & 24,40 & 0,17 & 1,50 \\
\hline & ve4 & 7,15 & 82,95 & 9,10 & 24,60 & 0,19 & 1,90 \\
\hline & ve5 & 7,10 & 83,50 & 9,05 & 23,50 & 0,23 & 1,70 \\
\hline & ve6 & 6,85 & 82,70 & 9,05 & 23,70 & 0,25 & 1,80 \\
\hline & ve7 & 6,90 & 84,30 & 8,95 & 23,70 & 0,50 & 2,30 \\
\hline \multirow{4}{*}{$\begin{array}{l}\text { Córrego da } \\
\text { Campininha }\end{array}$} & cal & 6,50 & 79,50 & 4,70 & 21,90 & 0,75 & 2,15 \\
\hline & $\mathrm{ca} 2$ & 6,50 & 69,00 & 5,10 & 21,60 & 0,30 & 1,23 \\
\hline & ca3 & 6,80 & 70,20 & 5,10 & 21,80 & 0,30 & 1,55 \\
\hline & $\mathrm{ca} 4$ & 6,90 & 70,60 & 5,30 & 21,70 & 1,80 & 5,40 \\
\hline \multirow{5}{*}{$\begin{array}{l}\text { Córrego } \\
\text { Itaguaraguaiaú }\end{array}$} & ch1 & 6,20 & 83,50 & 5,40 & 23,80 & 1,20 & 6,30 \\
\hline & $\operatorname{ch} 2$ & 6,30 & 84,20 & 5,50 & 23,40 & 0,80 & 5,40 \\
\hline & $\operatorname{ch} 3$ & 6,50 & 86,50 & 5,60 & 23,60 & 1,30 & 4,80 \\
\hline & $\operatorname{ch} 4$ & 6,20 & 88,20 & 5,40 & 22,80 & 0,80 & 3,20 \\
\hline & $\operatorname{ch} 5$ & 6,30 & 82,70 & 5,50 & 22,80 & 0,26 & 1,80 \\
\hline
\end{tabular}

O oxigênio dissolvido esteve acima de $5 \mathrm{mg} \mathrm{L}^{-1}$ em quase todos os pontos amostrados, evidenciando a boa oxigenação. O córrego da Campininha (ca) foi o que apresentou os menores valores de oxigênio dissolvido ficando entre 4,7 e 5,3. Apenas 1 ponto (ca1) entre todos os avaliados apresentou valor menor que 5,0 $\mathrm{mg} \mathrm{L}^{-1}$ (CONAMA $\mathrm{n}^{\mathrm{o}}$ 357/2005). Foram evidenciadas temperaturas altas no Rio Verde, Córrego da Campininha e o Parque Chico Mendes, com temperaturas de entre $21,6^{\circ} \mathrm{C}$ e $24,7^{\circ} \mathrm{C}$, e temperaturas menores nos rios 
Ribeirão do Ferro e Ipanema com temperaturas de no máximo $18,4^{\circ} \mathrm{C}$. Isso é explicado pela mata ripária mais conservada nesses dois últimos, que aumenta o sombreamento e diminui a temperatura mesmo nas épocas mais quentes.

Foi coletado um total de 522 indivíduos da espécie Melanoides tuberculata. No Rio Verde um total de 352 indivíduos foram capturados. O ponto ve1 foi o que apresentou a maior abundância de indivíduos e a maior degradação de suas margens. No córrego Itaguaraguaiaú foram coletados 135 indivíduos, no Ribeirão do Ferro, foram registrados 23 indivíduos enquanto que no Rio Ipanema foi obtida a menor abundância, um total de 10 indivíduos. $\mathrm{O}$ baixo número de indivíduos coletados no Rio Ipanema pode ser um indicativo de que a introdução de $M$. tuberculata nessa área seja recente, além de apresentar condições que podem não ser tão favoráveis para o desenvolvimento desta espécie. $\mathrm{O}$ córrego da Campininha foi o único local que não apresentou nenhum espécime (Figura 3).

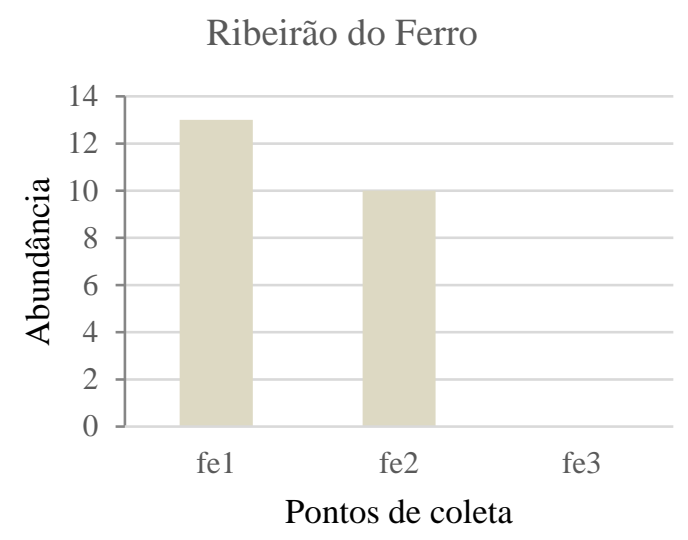

Rio Verde

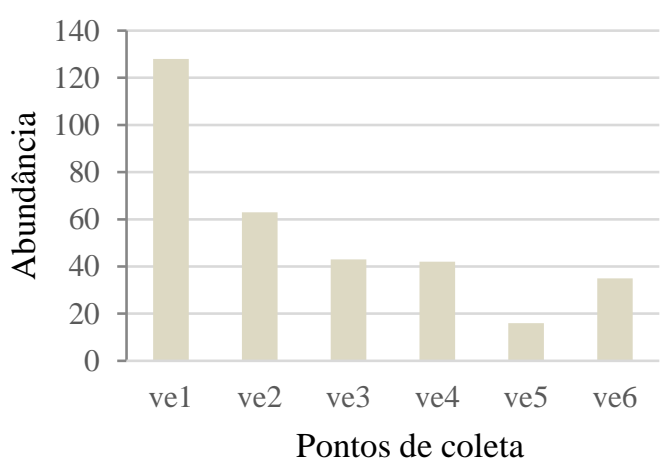

Rio Ipanema

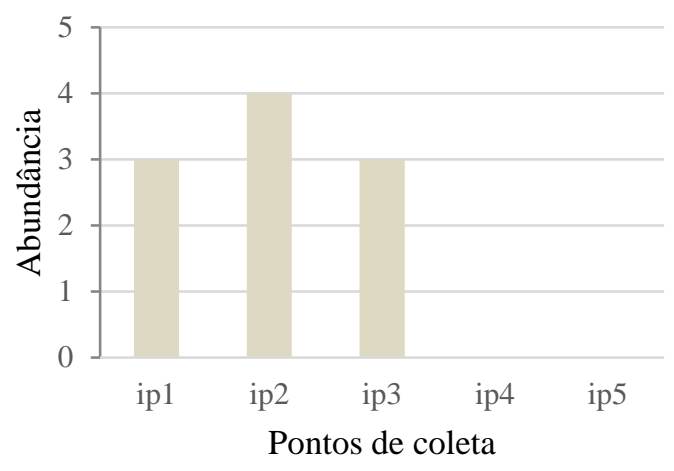

Córrego Itaguaraguaiaú

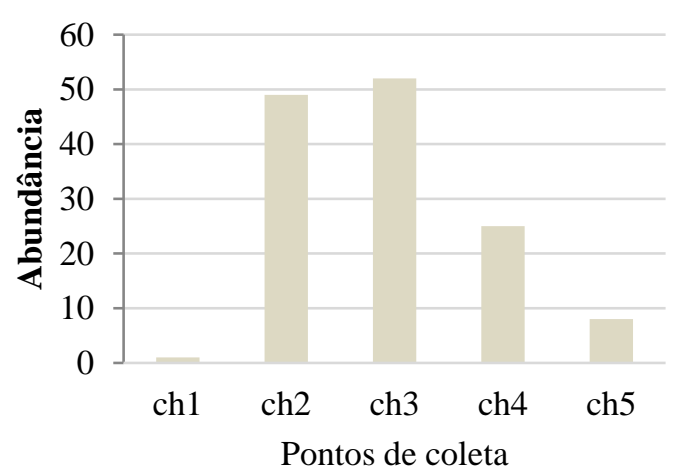

Figura 3. Abundância de M. tuberculata em cada um dos pontos amostrais.

De acordo com a Análise de Componentes Principais (Tabela 3) o eixo 1 foi forte e positivamente relacionado com substrato rochoso e mata conservada, e negativamente relacionado com condutividade, temperatura, substrato arenoso, mata degradada e abundância de $M$. tuberculata. No eixo 2 as variáveis $\mathrm{pH}$, oxigênio dissolvido, cascalho e abundância de $M$. tuberculata com valores positivos, e profundidade e largura com valores negativos foram as mais significativas. A ACP mostrou existir uma forte relação entre locais degradados e maiores abundâncias de $M$. tuberculata. Em resumo locais com substrato rochoso e mata ripária conservada apresentam baixa abundância da espécie, enquanto locais com altas temperaturas da água, com substrato arenoso e mata ripária degradada apresentam alta abundância de M. tuberculata. De Marco Junior (1999) e Martins-Silva e Barros (2001) afirmam ser sua característica marcante o fato de colonizar áreas impactadas por atividades 
antrópicas, principalmente locais de águas rasas, com substrato de granulometria fina e rico em detritos orgânicos. Outros autores como De Marco Junior et al. (1999) e De Sousa Souto et al. (2011) associam a ocorrência da M. tuberculata com áreas poluídas ou degradadas.

Tabela 3. Resultado da Análise de Componentes Principais: valores obtidos para os atributos em cada componente principal com as duas maiores porcentagens de variâncias.

\begin{tabular}{lcr}
\hline & PC 1 & PC 2 \\
\hline pH & $-0,10902$ & $\mathbf{0 , 4 7 1 0 8}$ \\
Condutividade & $\mathbf{- 0 , 3 8 0 5 8}$ & 0,027015 \\
Oxigênio dissolvido & 0,07681 & $\mathbf{0 , 3 5 0 2 6}$ \\
Temperatura & $\mathbf{0 , 4 2 9 3 7}$ & $-0,00694$ \\
Abundância & $\mathbf{0 , 2 5 6 2 8}$ & $\mathbf{0 , 1 0 9 0 3}$ \\
Areia & $\mathbf{0 , 1 8 3 2 3}$ & $\mathbf{- 0 , 2 4 6 7}$ \\
Cascalho & $\mathbf{- 0 , 1 5 8 3 3}$ & $\mathbf{0 , 2 3 5 4 6}$ \\
Rocha & $\mathbf{- 0 , 3 9 9 7 5}$ & $\mathbf{0 , 1 3 8 0 3}$ \\
Mata Degradada & $\mathbf{0 , 4 1 3 9 5}$ & 0,021127 \\
Mata Conservada & $\mathbf{- 0 , 4 1 3 9 5}$ & $-0,021127$ \\
Profundidade & 0,073154 & $\mathbf{- 0 , 5 4 0 7 1}$ \\
Largura & $-0,14432$ & $\mathbf{- 0 , 4 6 2 7 3}$ \\
\hline
\end{tabular}

Considerando o eixo 1 na análise de componentes principais, os pontos situados à esquerda correspondem aos mais conservados, apresentando correlação positiva com a condutividade, substrato rochoso e mata ripária conservada e negativa com abundância de $M$. tuberculata. A direita estão os pontos que apresentaram correlação positiva com substrato arenoso, mata ripária degradada, temperatura e abundância do gastrópode (Figura 4).

Os resultados mostraram que as características físicas e químicas dos sistemas aquáticos estudados contribuíram para a ocorrência e abundância da espécie. Isto porque por ser uma espécie altamente invasiva, uma característica marcante é sua capacidade de colonizar áreas impactadas por atividades antrópicas, principalmente locais de águas rasas, com substrato de granulometria fina e rico em detritos orgânicos (Martins-Silva e Barros, 2001; Okumura, 2006). Estudos realizados por Kock e Wolmarans (2009) também relacionaram a abundância de $M$. tuberculata em ambientes com substratos lamacentos ou arenosos e em temperaturas de $25^{\circ} \mathrm{C}$ em média. $M$. tuberculata é uma espécie muito tolerante e prolífica. Possui grande capacidade migratória e fácil adaptação, tornando-se bem estabelecida em todos os tipos de substrato e em corpos de água com diferentes graus de trofia e poluição (Suriani et al., 2007). Do Nascimento Filho et al. (2014) afirmam, que esse gastrópode ocorre em todos os tipos de ambientes (rios, lagos e reservatórios) e sua predominância pode ser explicada pelo fato de apresentar características como: abundância de jovens durante todo o ano, baixa taxa de mortalidade, alta capacidade migratória e de dispersão e a capacidade de se estabelecer, nos mais diversos substratos, o que corrobora com o encontrado pelo presente estudo. 


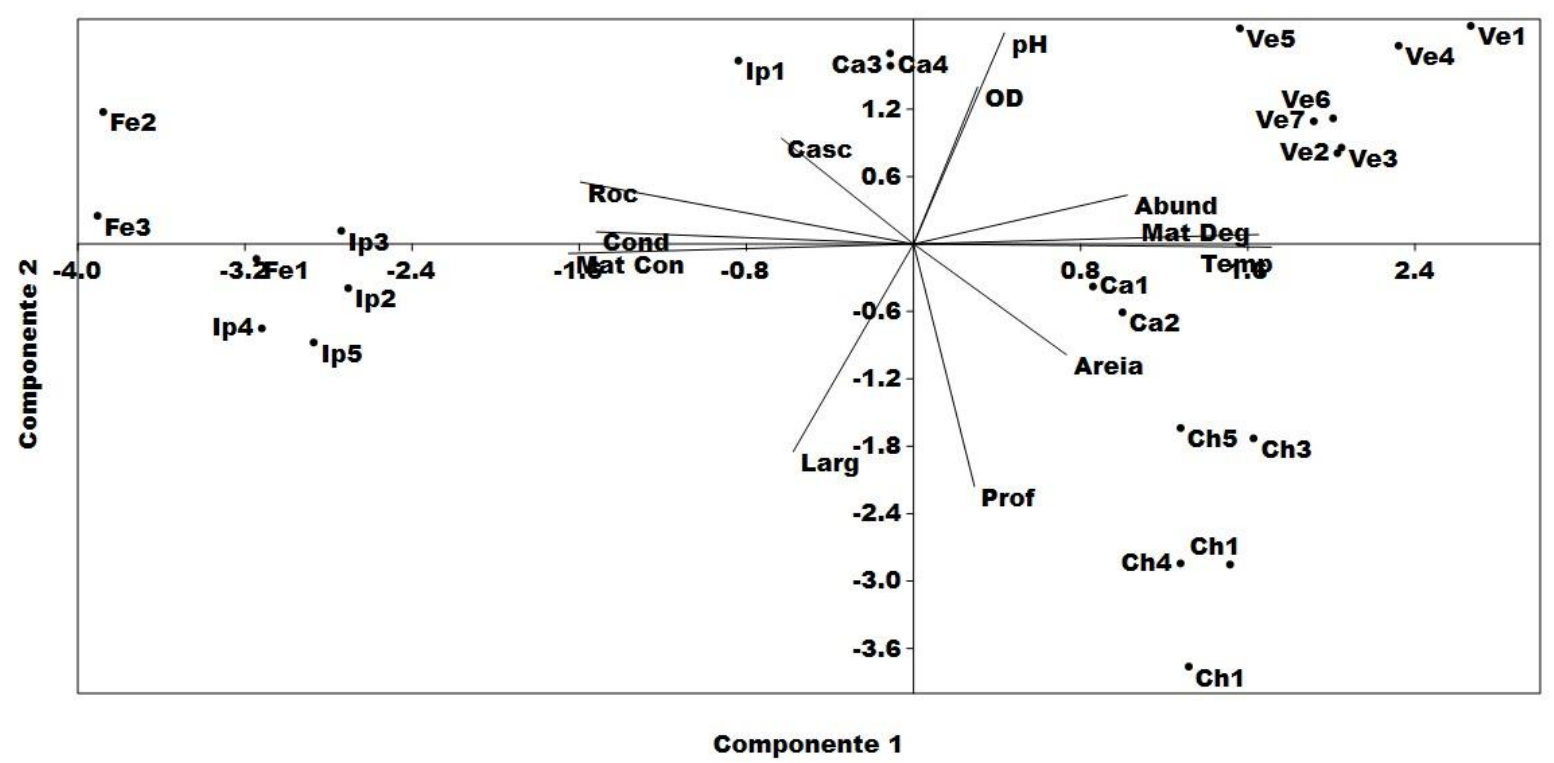

Figura 4. Análise de Componentes Principais, com os dados ambientais e abundância de Melanoides tuberculata em diferentes pontos amostrados.

O grande número de indivíduos encontrados no Rio Verde indica que a espécie encontra-se bem estabelecida no local. Não foram encontradas outras espécies de gastrópodes nos sete pontos amostrados, o que pode estar relacionado ao fato de que espécies invasoras exercem efeitos sobre as espécies nativas, sendo uma das principais causas da diminuição da biodiversidade, além de perda ou modificação de habitats nativos (Mack et al., 2000; Farani 2013). O número de indivíduos encontrados pode estar relacionado também ao fato de que as fêmeas dessa espécie podem se reproduzir por partenogênese, fato esse que agrava a proliferação desordenada e o torna um grande competidor (Farani, 2013), além de indicar também um provável impacto na comunidade bentônica como consequência da competição por alimento e por espaço (Lucca, 2003).

Fernandez et al. (2003) documentaram o declínio e o desaparecimento de algumas espécies de planorbídeos depois da introdução de $M$. tuberculata, o que pode explicar a presença apenas dessa espécie de molusco aquático em muitos dos pontos analisados por este estudo. Além disso, a maior abundância de M. tuberculata no Rio Verde pode ser devido ao maior impactado sofrido por este: ocupação urbana às margens, acentuada erosão, substrato formados predominantemente por areia, lançamento de esgoto, além de muitos pontos de assoreamento. É interessante a baixa abundância do molusco no córrego da Campininha, pois o mesmo apresenta alterações principalmente físicas devido ao assoreamento e a vegetação ripária. Possivelmente a restauração da vegetação que está sendo realizada atualmente e as medidas para conter a erosão deste córrego podem contribuir para que a espécie tenha dificuldade em colonizar o córrego. Abílio et al. (2007) afirmam que o fato de não ocorrer registro deste gastrópode em alguns locais estudados por eles está associado ao grau de isolamento desses corpos aquáticos e à não existência de um rio ou riacho que abasteça estes ambientes, uma vez que este gastrópode se encontra amplamente disperso.

A ocorrência de $M$. tuberculata em unidades de conservação da bacia hidrográfica do rio Sorocaba deve ser vista com preocupação e medidas devem ser tomadas para controle e erradicação. De Sousa Souto et al. (2011) também reforçam essa questão alertando que a ocorrência da espécie em duas áreas de conservação em Sergipe indica um sinal de alerta para os planos de gestão nestes ambientes. 


\section{CONSIDERAÇÕES FINAIS}

Estudos da malacofauna da bacia do Rio Sorocaba e o conhecimento das espécies invasoras são importantes ferramentas para que se possam traçar planos de ações visando o controle de $M$. tuberculata e a conservação da biodiversidade nativa da bacia. A ocorrência de $M$. tuberculata nas diferentes áreas estudadas, incluindo a zona de amortecimento da Flona de Ipanema e na própria unidade de conservação representa um sério risco às espécies nativas, podendo provocar perda de biodiversidade local, além da proliferação desta espécie a sistemas aquáticos conectados. Além disso, os resultados apresentados fornecem informações importantes da distribuição espacial de $M$. tuberculata e os principais fatores que regulam a sua abundância.

\section{AGRADECIMENTOS}

Os autores agradecem à Vice-Reitoria de Pós-Graduação e Pesquisa, UNIP, à Floresta Nacional de Ipanema (ICMBio) e à Prefeitura Municipal de Sorocaba, especialmente à Secretaria do Meio Ambiente, pelo apoio e auxilio no projeto.

\section{REFERÊNCIAS}

ABÍliO, F. J. P. Aspectos- bio-ecológicos da fauna malacológica, com ênfase a Melanoides turbeculata Müller, 1774 (Gastrópode, Thiaridae) em corpos aquáticos do Estado da Paraíba. 1997. 151 f. Dissertação (Mestrado) - Universidade Federal da Paraíba, João Pessoa, 1997.

ABÍllo, F. J. P.; WATANABE, T. Ocorrência de Lymnaea columella Say, 1817 (Gastropoda: Lymnaeidae), hospedeiro intermediário da Fasciola hepatica, para o Estado da Paraíba, Brasil. Revista de Saúde Pública, v. 32, n. 2, p. 185-186, 1998.

ABÍLIO, F. J. P.; RUFFO, T. L. de M.; DE SOUZA, A. H. F. F.; FLORENTINO, H. DA S.; OLIVEIRA JUNIOR, E. T. de; MEIRELES, B. N. et al. Macroinvertebrados bentônicos como bioindicadores de qualidade ambiental de corpos aquáticos da caatinga. Oecologia Australis, v. 11, n. 3, p. 397-409, 2007.

BARBOSA, F. S. Tópicos em Malacologia Médica. Rio de Janeiro: Fiocruz, 1995. 314 p.

DE MARCO JUNIOR, P. Invasion by the introduced aquatic snail Melanoides tuberculata (Müller, 1774) (Gastropoda: Prosobranchia: Thiaridae) of the Rio Doce State Park, Minas Gerais, Brazil. Studies on Neotropical Fauna and Environment, v. 34, n. 3, p. 186-189, 1999. https://doi.org/10.1076/snfe.34.3.186.8908

DE SOUSA SOUTO, L.; BRITO, M. F. G.; ROSA, L. C. da. Melanoides tuberculatus (Müller, 1774): a new threat to the conservation of native aquatic species in Sergipe, Brazil. Scientia Plena, v. 7, n. 4, 2011.

DIGBY, P. G. N.; KEMPTON, R. A. Multivariate Analysis of Ecological Communities. London: Chapman and Hall, 1987.

DO NASCIMENTO FILHO, S. L.; VIANA, G. F. S.; GOMES, R. L. M. Inventário da malacofauna límnica de três grandes reservatórios do sertão de Pernambuco, Brasil. Scientia Plena, v. 10, n. 11, p. 1-7, 2014.

EVERETT, R. A. Patterns and pathways of biological invasions. Trends in Ecology \& Evolution, v. 15, n. 5, p. 177-178, 2000. 
FARANI, G. L. Tolerância do gastrópode bioinvasor Melanoides tuberculatus (müller, 1774) à salinidade. 2013. 29f. Monografia (Bacharelado em Ciências Biológicas) Instituto de Biologia, Universidade Federal da Bahia, Salvador, 2013.

FERNADEZ, M. A.; THIENDO, S. C.; SIMONE, L. R. L. Distribution of the introduced freshwater snail Melanoides tuberculatus (Gastropoda: Thiaridae) in Brazil. The Nautilus, v. 117, n. 3, p. 78-82, 2003.

KOCK, K. N. DE; WOLMARANS, C. T. Distribution and habitats of Melanoides tuberculata (Müller, 1774) and M. victoriae (Dohrn, 1865) (Mollusca: Prosobranchia: Thiaridae) in South Africa. Water SA, v. 35, n. 5, p. 713-720, 2009. http://dx.doi.org/10.4314/ wsa.v35i5.49197

LUCCA, J. V. Análise comparativa da densidade e estrutura em tamanho de Melanoides tuberlata em quatro lagoas no Parque Estadual do Rio Doce, MG. In: CENTRO DE ECOLOGIA APLICADA, ESCOLA DE ENGENHARIA DE SÃO CARLOS. Workshop Impacto da Piscicultura e da introdução de espécies exóticas nas Bacias Hidrográficas. Itirapina, 2003.

MACK, R. N.; SIMBERLOFF, D.; LONSDALE, W. M.; EVANS, H.; ClOUT, M.; BAZZAZ, F. A. Biotic invasions: causes, epidemiology, global consequences, and control. Ecological Applications, v. 10, n. 3, p. 689-710, 2000. http://dx.doi.org/10.1890/1051-0761(2000)010[0689:BICEGC]2.0.CO;2

MANLY, B. J. Multivariate statistical methods: a primer. London: Chapman \& Hall, 1986. $281 \mathrm{p}$.

MARTINS-SILVA, M.; BARROS, M. Occurrence and distribution of freshwater molluscs in the Riacho Fundo CreekBasin, Brasília, Brazil. Revista de Biologia Tropical, v. 49, n. 3, p. 864-870, 2001.

OKUMURA, D. Estudos ecológicos e ecotoxicológicos de melanoides tuberculata, espécie exótica para a região neotropical. 2006. 159f. Dissertação (Mestrado) - Universidade de São Paulo, São Carlos, 2006.

PAULA-ANDRADE, C.; PINTO, H. A.; COSCARELLI, D.; VIDIGAL, T. H. D. A.; MELO, A. L. The natural infection of Melanoides tuberculata (Müller, 1774) (Mollusca: Gastropoda) by Centrocestus formosanus (Nishigori, 1924) (Platyhelminthes: Trematoda) in Paranoá lake, Brasília, Brazil. Brazilian Journal of Biology, v. 72, n. 2, p. 419-420, 2012. http://dx.doi.org/10.1590/S1519-69842012000200026

PAMPLIN, P. A. Z.; ROCHA, O.; MARCHESE, M. Riqueza de espécies de Oligochaeta (Annelida, Clitellata) em duas represas do rio Tietê (São Paulo). Biota Neotropica, v. 5, n. 1, p. 63-70, 2005. http://dx.doi.org/10.1590/S1676-06032005000100007

PAULA-ANDRADE, C.; PINTO, H. A.; COSCARELLI, D.; VIDIGAL, T. H. D. A.; MELO, A. L. The natural infection of Melanoides tuberculata (Müller, 1774) (Mollusca: Gastropoda) by Centrocestus formosanus (Nishigori, 1924) (Platyhelminthes: Trematoda) in Paranoá lake, Brasília, Brazil. Brazilian Journal of Biology, v. 72, n. 2, p. 419-420, 2012. http://dx.doi.org/10.1590/S1519-69842012000200026

PIMENTEL, D.; MCNAIR, S.; JANECKA, J.; WIGHTMAN, J.; SIMMONDS, C.; O'CONNELL, C.et al. Economic and environmental threats of alien plant, animal, and microbe invasions. Agriculture, Ecosystems \& Environment, v. 84, p. 1-20, 2001. https://doi.org/10.1016/S0167-8809(00)00178-X 
PINTO, H. A.; MELO, A. L. de. Melanoides tuberculata (Mollusca: Thiaridae) as an intermediate host of Centrocestus formosanus (Trematoda: Heterophyidae) in Brazil. Revista do Instituto de Medicina Tropical de São Paulo, v. 52, n. 4, p. 207-210, 2010. http://dx.doi.org/10.1590/S0036-46652010000400008

ROCHA, O.; ESPINDOLA, E. L. G.; RIETZLER, A. C.; FENERIC-VERANI, N.; VERANI, J. R. Animal invaders in São Paulo state reservoirs. Oecologia Australis, v. 15, n. 3, p. 631-642, 2011. http://dx.doi.org/10.4257/oeco.2011.1503.14

ROCHA-MIRANDA, F.; MARTINS-SILVA, M. J. Primeiro registro do gastrópode africano invasor Melanoides tuberculatus (Gastropoda: Prosobranchia: Thiaridae) na Bacia do Rio Paranã, GO, Brasil. Brazilian Journal of Biology, v. 66, n. 4, p. 1109-1115, 2006. http://dx.doi.org/10.1590/S1519-69842006000600018

ROSA, L. C.; SOUTO, L. S.; BRITO, M. F. Ocorrência do molusco exótico invasor Melanoides tuberculatus na Lagoa Azul, APA Litoral Sul, Sergipe, Brasil. In: ENCONTRO DE RECURSOS HIDRICOS EM SERGIPE, 3., 24-26 mar. 2010, Aracaju. Trabalhos... Aracaju: SRH; EMBRAPA Tabuleiros Costeiros, 2010. 4p.

SANTOS, C. M.; ESKINAZI-SANT'ANNA, E. M. The introduced snail Melanoides tuberculatus (Muller, 1774) (Mollusca: Thiaridae) in aquatic ecosystems of the Brazilian Semiarid Northeast (Piranhas-Assu River basin, Stateof Rio Grande do Norte). Brazilian Journal of Biology, v. 70, n. $1,2010$. http://dx.doi.org/10.1590/S1519-69842010000100003

SÃO PAULO. Secretaria do Meio Ambiente Espécies exóticas invasoras: proposta de estratégia para abordar a questão. São Paulo, 2009.

SILVA. E. C. da; BARROS, F. Macrofauna bentônica introduzida no brasil: lista de espécies marinhas e dulcícolas e distribuição atual. Oecologia Australis, v. 15, n. 2, p. 326-344, 2011. http://dx.doi.org/10.4257/oeco.2011.1502.10

SMITH, W. S. Os peixes do Rio Sorocaba: a história de uma bacia hidrográfica. Sorocaba: TCM Comunicação, 2003. 164 p.

SOUZA, C. P.; LIMA, L. C. Moluscos de interesse parasitológico do Brasil. 2. ed. Belo Horizonte: Fundação Oswaldo Cruz; Centro de Pesquisas René Rachou, 1997. 79 p.

SOUZA, M. A. A.; BARBOSA, V. S.; JONES, O. A.; BOCANEGRA, S.; SOUZASANTOS, R.; PAREDES, H. et al. Aspectos ecológicos e levantamento malacológico para identificação de áreas de risco para transmissão da esquistossomose mansoni no litoral norte de Pernambuco, Brasil. Iheringia, Série Zoológica, v. 100, n. 1, p. p. 19$24,2010$.

SOUZA, M. A. A.; SOUZA, L. A.; MACHADO-COELHO, G. L. L.; MELO, A. L. Levantamento malacológico e mapeamento das áreas de risco para transmissão da esquistossomose mansoni no município de Mariana, Minas Gerais, Brasil. Revista de Ciências Médicas e Biológicas, v. 5, n. 2, p. 132-139, 2006. http://dx.doi. org/10.9771/cmbio.v5i2.4120

SURIANI, A. L.; FRANÇA, R. S.; ROCHA, O. A malacofauna bentônica das represas do médio rio Tietê (São Paulo, Brasil) e uma avaliação ecológica das espécies exóticas invasoras, Melanoides tuberculata (Müller) e Corbicula flumínea (Müller). Revista Brasileira de Zoologia, v. 24, p. 21-32, 2007. 
THIENGO, S. C.; FERNANDEZ, M. A.; MATTOS, A. C.; BARBOSA, A. F. Dispersão do molusco introduzido Melanoides tuberculatus (Muller, 1774) (Gastropoda: Thiaridae) no Brasil. Tópicos em Malacologia-Ecos do XVIII Encontro Brasileiro de Malacologia. Rio de Janeiro: Sociedade Brasileira de Malacologia, 2007. p. 101-106.

VAZ, J. F.; TELES, H. M. S.; CORREA, M. A.; LEITE, S. P. S. Ocorrência no Brasil de Thiara (Melanoides tuberculatus) Müller, 1774, (Gastropoda, Prosobranchia), primeiro hospedeiro intermediário de Clonorchissinensis (Cobbold, 1875) (Trematoda, Plathyhelmintes). Revista de Saúde Pública, v. 20, n. 4, p. 318-322, 1986. http://dx.doi.org/10.1590/S0034-89101986000400008

WELCOMME, R. L. International introductions of Inland aquatic species. Rome: FAO, 1988. 318 p. (Fisheries Paper, no 294) 